\title{
ENVAHISSEMENT GANGLIONNAIRE CERVICAL DANS LES CANCERS DU LARYNX
}

\author{
I. CHARFEDDINE, J. MOALLA, M. BEN AMOR, N. REKHIS, N. KHOUFI, N. FEKI,
} A. CHAKROUN, A. GHORBEL

SERVICE ORL ET DE CHIRURGIE CERVICO-FACIALE CHU HABIB BOURGUIBA. SFAX.

\section{INTRODUCTION}

\section{RESUME}

Le but de notre travail est d'étudier la fréquence de l'atteinte ganglionnaire dans le cancer du larynx, les facteurs influençant cette atteinte et d'en évaluer la valeur pronostique.

MALADES ET METHODES : Notre étude est rétrospective, colligée sur une période de 9 ans, portant sur 105 cas de cancer du larynx ayant bénéficié d'une chirurgie ganglionnaire. L'âge moyen était de 61,8 ans. La prédominance masculine était très nette. L'évidement ganglionnaire conservateur fonctionnel bilatéral était réalisé dans la majorité des cas (87,6\%).

RESULTATS : L'envahissement ganglionnaire histologique global était de $35,2 \%$ des cas. II était multiple dans $73 \%$. Le taux de rupture capsulaire était de $40 \%$. Les secteurs II et III étaient les territoires les plus intéressés.

Le taux d'envahissement histologique ganglionnaire était plus fréquent en cas de présence d'adénopathie palpable et pour les types peu ou moyennement différenciés.

L'étude statistique a montré que de mauvais pronostic, étaient l'atteinte ganglionnaire palpable, l'envahissement ganglionnaire histologique multiple et surtout la présence de rupture capsulaire.

\section{CONCLUSION}

Le taux de métastase ganglionnaire occulte varie de 17,6 à $30 \%$ des cas avec rupture capsulaire variant de 10 à $50 \%$ des cas.

Les taux de métastases ganglionnaires histologiques $\left(N_{+}\right)$et de rupture capsulaire $(R+)$ augmentent avec le stade " $N$ " clinique.

Enfin, la présence de rupture capsulaire et le nombre de ganglions envahis affectent de façon significative la survie. MOTS CLÉs : Cancer du larynx - envahissement ganglionnaire - pronostic

\section{OBJECTIVE}

\section{SUMMARY}

The aim of our study is to study the frequency of lymph node metastases in laryngeal cancer, and to analyze factors affecting this lymphatic involvement and his prognosis.

\section{PATIENTS AND METHODES}

It's a retrospective study about 105 cases of laryngeal cancer, colliged over a period of 9 years and treated by surgery. Mean age was 61.8 years. Bilateral conservative neck dissection was performed in the most cases $(87.6 \%)$.

RESULTS: Cervical nodes were histologically involved in $35.2 \%$ of cases. The incidence of extracapsular spread was $40 \%$ of cases. The levels II and III were the most common sites of metastases.

Histologic evidence of lymph nodes metastases were correlated to the importance of palpable lymph nodes and the histologic type of tumor.

Statistical study had showen that the presence of palpable lymph nodes, histologic metastasis and extracapsular spread were associated with poorer prognosis.

\section{CONCLUSION}

The reported incidence of occult lymph nodes metastases ranges from 17.6 to $30 \%$ of cases. Histologic lymph node metastases and extracapsular spread are correlated to clinical stage.

Extracapsular spread and the number of lymph nodes metastasis are the most important factor in mortality. KEY WORDS: Laryngeal cancer - lymph node metastases - prognosis

\section{INTRODUCTION}

En Tunisie, le cancer du larynx occupe la $2^{\text {ime }}$ place parmi les cancers des voies aérodigestives supérieures après celui du cavum. Sa lymphophilie est connue et constitue- rait le facteur déterminant significatif dans l'évolution. Dans ce travail, nous nous sommes proposés de répondre à trois questions :

- Quelle est la fréquence de l'atteinte ganglionnaire dans 
le cancer du larynx?

- Y a-t-il des facteurs cliniques et histologiques, locaux et régionaux influençant cette atteinte?

- S'agit-il d'un facteur déterminant dans l'évolution de ces cancers?

\section{MATÉRIEL ET MÉTHODES}

Notre étude est rétrospective, menée sur une période de 9 ans (1994-2002) à propos de 105 cas de cancer de larynx ayant bénéficié d'un traitement chirurgical de la tumeur laryngée et des aires ganglionnaires cervicales. L'âge moyen de nos malades était de 61,8 ans ( 37-90 ans) avec une prédominance masculine nette.

Une laryngectomie totale avec l'évidement ganglionnaire cervical (EC) fonctionnel bilatéral étaient réalisé dans la majorité des cas. (Tableau l et II).

La radiothérapie post opératoire des aires ganglionnaires cervicales était préconisée en cas d'envahissement histologique de plus d'un ganglion. Elle se donnait à la dose de 50 Gy selon un étalement classique.

\begin{tabular}{|l|c|c|}
\hline \multicolumn{1}{|c|}{ Gestes } & Nombre & $\%$ \\
\hline EC fonctionnel Bilatéral & 92 & 87,6 \\
\hline $\begin{array}{l}\text { EC radical Homolatéral + } \\
\text { EC fonctionnel controlatéral }\end{array}$ & 7 & 6,7 \\
\hline $\begin{array}{l}\text { EC radical Modifié + EC } \\
\text { fonctionnel }\end{array}$ & 6 & 5,7 \\
\hline EMR & 23 & 21,9 \\
\hline EC submandibulaire & 9 & 8,6 \\
\hline
\end{tabular}

EC: Evidement ganglionnaire cervical EMR: Evidement médiasti no-récurrentiel

Tableau I : Types d'évidement ganglionnaire

\begin{tabular}{|l|c|c|}
\hline \multicolumn{1}{|c|}{ Types de chirurgie } & Nombre & $\%$ \\
\hline LT & 70 & 66,6 \\
\hline Hémi-pharyngo LT & 6 & 5,7 \\
\hline Sub glosso LT & 13 & 12,4 \\
\hline LT carrée & 4 & 3,8 \\
\hline L sub totale & 12 & 11,5 \\
\hline
\end{tabular}

LT:Lar yngectomie totale

Tableau II : Traitement de la tumeur primitive

\section{RESULTATS}

L'adénopathie cervicale palpable était rarement trouvée au premier examen : 16 cas, soit $15,2 \%$ des cas et plus rarement révélatrice $(7,6 \%)$. Le siège jugulo-carotidien haut représentait la localisation la plus fréquente ( $87 \%$ des cas).

Les adénopathies étaient homolatérales à la tumeur primitive dans $68,7 \%$ des cas et souvent unique $(75 \%$ des cas).

L'examen anatomopathologique définitif a montré la présence d'envahissement ganglionnaire histologique $(\mathrm{N}+)$ dans $35,2 \%$ des cas. L'atteinte était multiple dans $73 \%$ des cas. Le taux de rupture capsulaire était de $40 \%$ et de métastases ganglionnaires occultes de $27 \%$. L'atteinte ganglionnaire était essentiellement soit homolatérale à la tumeur primitive $\quad(46 \%)$ soit bilatérale $(46 \%)$.

Les secteurs II et III étaient les sites les plus souvent intéressés, que ce soit du côté homolatéral ou controlatéral à la tumeur. Le territoire sus claviculaire était envahi de façon moins fréquente : $14,3 \%$ des cas (tableau III).

\begin{tabular}{|l|c|c|c|}
\hline \multicolumn{1}{|c|}{ Secteurs } & $\%$ & Atteinte Homolatérale & Atteinte Bilatérale \\
\hline II & 29,5 & $20 \%$ & $9,5 \%$ \\
\hline III & 24,7 & $15,2 \%$ & $9,5 \%$ \\
\hline IV & 14,3 & $11,4 \%$ & $2,9 \%$ \\
\hline VI & 9,6 & $6,7 \%$ & $2,9 \%$ \\
\hline I & 2,9 & $1,9 \%$ & $1 \%$ \\
\hline
\end{tabular}

Tableau III : Sites ganglionnaires atteints $(\mathrm{N}+)$

L'étude des facteurs influençant l'envahissement ganglionnaire a montré que :

- Le taux d'envahissement ganglionnaire histologique était plus important en cas de présence d'adénopathie palpable qu'en cas de NO clinique ( $\mathrm{NO}=27 \%$ $\mathrm{Np}=81,3 \%$ ).

- Il était aussi plus important pour les types histologiques peu ou moyennement différenciés $(53 \%)$ que pour le type histologique bien différencié $(30 \%)$ avec des tests statistiques significatifs $(p<0,05)$.

- Le stade tumoral « $T$ » n'interférait pas dans le risque d'envahissement ganglionnaire ( T2 :33,4\%; T3 : 34,5\% et $\mathrm{T} 4: 37,8 \%$ )

- Par contre, la localisation de l'envahissement ganglionnaire était en corrélation statistiquement significative avec le siège de la tumeur primitive (Tableau IV).

\begin{tabular}{|l|c|c|c|c|c|c|c|c|c|c|c|c|}
\hline & \multicolumn{9}{|c|}{ Côté homolatéral } & \multicolumn{6}{|c|}{ Côté controlatéral } \\
\cline { 2 - 13 } & I & II & $\begin{array}{c}\text { II } \\
\text { Bis }\end{array}$ & III & IV & VI & I & II & $\begin{array}{c}\text { II } \\
\text { Bis }\end{array}$ & III & IV & VI \\
\hline $\begin{array}{l}\text { Sus glottique ou } \\
\text { glotto-sus- } \\
\text { glottique (40 cas) }\end{array}$ & NF & 18,4 & 10,5 & 16 & 10,5 & 5,2 & $\mathbf{N F}$ & $\mathbf{1 0 , 5}$ & $\mathbf{2 , 6}$ & $\mathbf{5 , 3}$ & $\mathbf{0}$ & $\mathbf{0}$ \\
\hline $\begin{array}{l}\text { Glotto- } \\
\text { sousglottique } \\
\text { (13cas) }\end{array}$ & NF & 0 & 0 & 7,6 & 7,6 & 0 & NF & $\mathbf{0}$ & $\mathbf{0}$ & $\mathbf{0}$ & $\mathbf{0}$ & $\mathbf{0}$ \\
\hline 3 étages ( 52 cas) & 2,9 & 18 & 2 & 17,7 & 15,5 & 5,5 & $\mathbf{0}$ & $\mathbf{6 , 6}$ & $\mathbf{4}$ & $\mathbf{1 5 , 5}$ & $\mathbf{4 , 4}$ & $\mathbf{0}$ \\
\hline NF : Non fait
\end{tabular}

Tableau IV : Secteurs envahis en fonction du siège de la tumeur (\%).

La bilatéralité de l'envahissement ganglionnaire était plus fréquente dans les tumeurs sus glottiques et des 3 étages.

Concernant la valeur pronostique de l'atteinte ganglionnaire, l'étude statistique a montré que de mauvais pronostic étaient l'atteinte ganglionnaire palpable, l'envahissement histologique de plusieurs ganglions au niveau de 
plusieurs secteurs et la présence de rupture capsulaire. (Tableau V).

\begin{tabular}{|l|l|l|c|}
\hline Paramètres & \multicolumn{2}{|c|}{ Survie à 3 ans } & P \\
\hline N.Clinique & N0 & $86 \%$ & 0,0005 \\
& N1-N2 & $50 \%$ & \\
\hline N.Histologique & $\mathrm{N}-$ & $90 \%$ & $<0,05$ \\
& $\mathrm{~N}+<2$ & $86 \%$ & \\
& $2<\mathrm{N}+<5$ & $75 \%$ & $<0,05$ \\
& $\mathrm{~N}+>5$ & $33 \%$ & $88 \%$ \\
\hline Rupture Capsulaire & $\mathrm{R}-$ & $48 \%$ & \\
& $\mathrm{R}+$ & & \\
\hline
\end{tabular}

Tableau V : Impact de l'atteinte ganglionnaire sur la survie.

\section{DISCUSSION}

Le taux de métastases ganglionnaires occultes varie de 17,6 à $30 \%$ des cas $(1,2,3)$. II était de $27 \%$ dans notre série.

Le taux de rupture capsulaire varie dans la littérature entre 10 et $50 \%(1,2,4,5)$. II était de $40 \%$ dans notre série. La fréquence de l'atteinte ganglionnaire est variable d'un secteur à l'autre $(5,6,7)$, ( tableau VI).

\begin{tabular}{|l|c|c|c|c|}
\hline \multicolumn{1}{|c|}{ Auteurs } & Ming & Buckley & Moe & Notre série \\
\hline Secteur & & & & \\
\hline I & 1,36 & 0,2 & 6,5 & 2,9 \\
\hline II & 27,4 & 22,8 & 6,5 & 29,5 \\
\hline III & 39,7 & 9,7 & 25,8 & 24,7 \\
\hline IV & 15 & 3,8 & 12,9 & 14,3 \\
\hline V & 5,4 & 0 & 7,5 & NF \\
\hline VI & NS & NS & NS & 9,6 \\
\hline NF : Non fait & \multicolumn{2}{|c|}{ NS : Non signalé }
\end{tabular}

Tableau VI : Envahissement ganglionnaire selon es secteurs (\%).

L'étude des facteurs influençant l'atteinte ganglionnaire a montré que l'incidence de métastases ganglionnaires et de rupture capsulaire varie en fonction de la taille de l'adénopathie palpée et de son stade « $N$ ». $(5,8,9,10,12)$ (Tableau VII).

\begin{tabular}{|l|cc|cc|cc|}
\hline \multirow{2}{*}{ Auteurs N } & \multicolumn{2}{|c|}{ N0 } & \multicolumn{2}{c|}{ N1 } & \multicolumn{2}{|c|}{ N2-N3 } \\
\hline & N+ & R+ & N+ & R+ & N+ & R+ \\
\hline Nancy & 40 & 20 & 82 & 38 & 93 & 67 \\
\hline Richard & 50 & 19 & 60 & 35 & 83 & 50 \\
\hline Ming & 32 & 11 & 80 & 24 & 91 & 64 \\
\hline Notre série & 27 & 9 & 67 & 17 & 90 & 66 \\
\hline
\end{tabular}

Tableau VII : Envahissement ganglionnaire selon le stade $\mathbf{N}(\%)$.

L'envahissement ganglionnaire est variable aussi selon le siège de la tumeur au niveau du larynx. Le siège sus-glottique est le plus lymphophile et l'incidence des métastases ganglionnaires des cancers sus-glottiques varie de 38,5 à $65 \%$. Lès cáncers multi-régionaux ont aussi un risque élevé de métastases ganglionnaires variant de 40 à $45 \%(4,7,10)$.
Pour la majorité des auteurs $(1,9,11)$, le stade « $T$ » n'a pas de valeur prédictive du risque d'envahissement ganglionnaire.

Quant au grade histologique, pour certains auteurs (9), il n'est pas un facteur prédictif de risque de métastases ganglionnaires. Cependant, d'après l'étude de Laccourey (11) et cette étude, les cancers moins différenciés sont plus lymphophiles que les cancers bien différenciés avec une différence statistiquement significative.

Concernant la valeur pronostique de l'atteinte ganglionnaire, la présence d'envahissement ganglionnaire histologique $\mathrm{N}+$ et/ ou de rupture capsulaire $\mathrm{R}+$ affecte la survie de façon significative $(8,9)$ (tableau VIII).

\begin{tabular}{|l|c|c|c|}
\hline $\mathrm{N}$ hist /Auteurs & Richard & Nancy & Notre série \\
\hline $\mathrm{N}-$ & 63 & 73 & 90 \\
\hline $\mathrm{N}+\mathrm{R}-$ & 46 & 79 & 88 \\
\hline $\mathrm{N}+\mathrm{R}+$ & 19 & 45 & 48 \\
\hline $\mathrm{P}$ & $<0,0001$ & $<0,001$ & $<0,05$ \\
\hline
\end{tabular}

Envahissement ganglionnaire histologique : $\mathrm{N}+$

Rupture capsulaire: R+

Tableau VIII : Valeur pronostique (Survie à 3ans).

impact dans la diminution du taux de survie. En effet la survie à 4 ans chute de 80 à $20 \%$ lorsque le nombre de ganglions envahis passe de moins de 2 à plus que 4 (12).

\section{CONCLUSION}

L'envahissement ganglionnaire est assez fréquent. II a un impact sur la survie.

Le traitement ganglionnaire s'impose. Le meilleur est le traitement chirurgical qui permet une classification histologique ganglionnaire et aussi de planifier une radiothérapie post opératoire. Mais quel type de traitement faut-il préconiser?

C'est l'évidement ganglionnaire emportant au moins les secteurs II et III (2).

Enfin, il est intéressant de justifier la théorie du ganglion sentinelle pour éviter une chirurgie étendue et non nécessaire pour les cous No. 


\section{REFERENCES}

1- S. Hosal, RL. Carrau, JT. Johnson, en. Myers. Selective neck dissection in the management of the clinically node negative neck. Laryngoscope. 2000; 110:2037-2040.

2- X. Leon, M. Quer, C. Orus, F. Jose Sancho et Al. Selective dissection of levels II-III with intraoperative control of the upper and middle jugular nodes: a therapeutic option for the N0 neck. Head Neck 2001: 441-446.

3- G. Spiano, R. Piantanida, R. Pellini, L. Muscatello. Elective treatment of the neck in squamous cell carcinoma of the larynx: clinical experience. Head Neck February 2003: 97-102.

4- J. Buckley, Ken Maclennan. Cervical node metastases in laryngeal and hypopharyngeal cancer: a prospective analysis of prevalence and distribution. Head Neck 2000: 380-385.

5 - X. Ming et Coll. Cervical lymph node metastatic patterns of squamous carcinomas in the upper aerodigestive tract. JF ORL . 1996; 110:937-941.

6 - T. Buckley, T. Feber. Surgical treatement of cervical node metastases from squamous carcinoma of the upper aerodigestive tract: evaluation of the evidence for modifications of neck dissection. Head Neck. 2001:907-915.

7- K. Moe, GT. Wolf, SG. Fisher, WK. Hong. Regional metastases in patients with advanced laryngeal cancer. Departement of Veterans Affairs laryngeal cancer study group. Arch oto laryngeal Head Neck surg. 1996;122:644-648. 8- L. Nancy. Extracapsular spread of carcinoma in cervical lymph nodes. Impact upon survival in patients with carcinoma of the supraglottic larynx. Cancer 1985,56:1597-1599.

9- JM. Richard, H. Sancho-garnier, C. Micheau, C. Cachin. Prognostic factors of cervical lymph nodes metastasis in upper respiratory and digestive tract carcinomas.Laryngoscope.1987; 97:97-101.

10- F. Marlier et Coll. Apport de l'imagerie dans le bilan ganglionnaire des cancers du larynx N0. Cah ORL - T.XXXVI, 1 : 29-37.

11- H. Laccourrey, P. Beutter, J. Lacau Saint-guily.. Les adénopathies des épithéliomas du larynx. Fréquence et pronostic. Ann. Otol Laryngol. 1979 ;96(6): 329-348.

12- CJ. O'brien. Neck dissection with and without radiotherapy: prognostic factors, patterns of recurrence, and survival. Am J surgery. 1986; 152:456463 